\title{
Statistical Mechanics of Online Learning for Ensemble Teachers
}

\author{
Seiji Miyoshi, Member, IEEE, and Masato Okada
}

\begin{abstract}
We analyze the generalization performance of a student in a model composed of linear perceptrons: a true teacher, ensemble teachers, and the student. Calculating the generalization error of the student analytically using statistical mechanics in the framework of online learning, we prove that when the learning rate satisfies $\eta<1$, the larger the number $K$ is and the more variety the ensemble teachers have, the smaller the generalization error is. On the other hand, when $\eta>1$, the properties are completely reversed. If the variety of the ensemble teachers is rich enough, the direction cosine between the true teacher and the student becomes unity in the limit of $\eta \rightarrow 0$ and $K \rightarrow \infty$.
\end{abstract}

\section{INTRODUCTION}

Learning is to infer the underlying rules that dominate data generation using observed data. Observed data are inputoutput pairs from a teacher and are called examples. Learning can be roughly classified into batch learning and online learning [1]. In batch learning, given examples are used more than once. In this paradigm, a student becomes to give correct answers after training if the student has adequate freedom. However, it is necessary to have a long amount of time and a large memory in which to store many examples. On the contrary, in online learning examples once used are discarded. In this case, a student cannot give correct answers for all examples used in training. However, there are merits, for example, a large memory for storing many examples isn't necessary, and it is possible to follow a time variant teacher.

Recently, we [5], [6] analyzed the generalization performance of ensemble learning [2], [3], [4] in a framework of online learning using a statistical mechanical method [1], [8]. Using the same method, we also analyzed the generalization performance of a student supervised by a moving teacher that goes around a true teacher[7]. As a result, it was proven that the generalization error of a student can be smaller than a moving teacher, even if the student only uses examples from the moving teacher. In an actual human society, a teacher observed by a student does not always present the correct answer. In many cases, the teacher is learning and continues to change. Therefore, the analysis of such a model is interesting for considering the analogies between statistical learning theories and an actual human society.

On the other hand, in most cases in an actual human society a student can observe examples from two or more

Seiji Miyoshi is with the Department of Electronic Engineering, Kobe City College of Technology, 8-3 Gakuen-higashimachi, Nishi-ku, Kobe-shi, 651-2194 Japan (phone: +81-78-795-3247; fax: +81-78-795-3314; email: miyoshi@kobe-kosen.ac.jp).

Masato Okada is with the Division of Transdisciplinary Sciences, Graduate School of Frontier Sciences, The University of Tokyo, 5-1-5 Kashiwanoha, Kashiwa-shi, Chiba, 277-8561 Japan, RIKEN Brain Science Institute, 2-1 Hirosawa, Wako-shi, Saitama, 351-0198 Japan, and JST PRESTO, 5-1-5 Kashiwanoha, Kashiwa-shi, Chiba, 277-8561 Japan. teachers who differ from each other. Therefore, we analyze the generalization performance of such a model and discuss the use of imperfect teachers in this paper. That is, we consider a true teacher and $K$ teachers called ensemble teachers who exist around the true teacher. A student uses input-output pairs from ensemble teachers in turn or randomly. In this paper, we treat a model in which all of the true teacher, the ensemble teachers and the student are linear perceptrons[5] with noises. We obtain order parameters and generalization errors analytically in the framework of online learning using a statistical mechanical method. As a result, it is proven that when student's learning rate satisfies $\eta<1$, the larger the number $K$ is and the more variety the ensemble teachers have, the smaller the student's generalization error is. On the other hand, when $\eta>1$, the properties are completely reversed. If the variety of ensemble teachers is rich enough, the direction cosine between the true teacher and the student becomes unity in the limit of $\eta \rightarrow 0$ and $K \rightarrow \infty$.

\section{MODEL}

In this paper, we consider a true teacher, $K$ ensemble teachers and a student. They are all linear perceptrons with connection weights $\boldsymbol{A}, \boldsymbol{B}_{k}$ and $\boldsymbol{J}$, respectively. Here, $k=$ $1, \ldots, K$. For simplicity, the connection weight of the true teacher, the ensemble teachers and the student are simply called the true teacher, the ensemble teachers and the student, respectively. True teacher $\boldsymbol{A}=\left(A_{1}, \ldots, A_{N}\right)$, ensemble teachers $\boldsymbol{B}_{k}=\left(B_{k 1}, \ldots, B_{k N}\right)$, student $\boldsymbol{J}=\left(J_{1}, \ldots, J_{N}\right)$ and input $\boldsymbol{x}=\left(x_{1}, \ldots, x_{N}\right)$ are $N$ dimensional vectors. Each component $A_{i}$ of $\boldsymbol{A}$ is drawn from $\mathcal{N}(0,1)$ independently and fixed, where $\mathcal{N}(0,1)$ denotes Gaussian distribution with a mean of zero and variance unity. Some components $B_{k i}$ are equal to $A_{i}$ multiplied by -1 , the others are equal to $A_{i}$. Which component $B_{k i}$ is equal to $-A_{i}$ is independent from the value of $A_{i}$. Hence, $B_{k i}$ also obeys $\mathcal{N}(0,1) . B_{k i}$ is also fixed. The direction cosine between $\boldsymbol{B}_{k}$ and $\boldsymbol{A}$ is $R_{B k}$ and that between $\boldsymbol{B}_{k}$ and $\boldsymbol{B}_{k^{\prime}}$ is $q_{k k^{\prime}}$. Each of the components $J_{i}^{0}$ of the initial value $\boldsymbol{J}^{0}$ of $\boldsymbol{J}$ is drawn from $\mathcal{N}(0,1)$ independently. The direction cosine between $\boldsymbol{J}$ and $\boldsymbol{A}$ is $R_{J}$ and that between $\boldsymbol{J}$ and $\boldsymbol{B}_{k}$ is $R_{B k J}$. Each component $x_{i}$ of $\boldsymbol{x}$ is drawn from $\mathcal{N}(0,1 / N)$ independently. 
Thus,

$$
\begin{aligned}
\left\langle A_{i}\right\rangle & =0, \quad\left\langle\left(A_{i}\right)^{2}\right\rangle=1, \\
\left\langle B_{k i}\right\rangle & =0, \quad\left\langle\left(B_{k i}\right)^{2}\right\rangle=1 \\
\left\langle J_{i}^{0}\right\rangle & =0, \quad\left\langle\left(J_{i}^{0}\right)^{2}\right\rangle=1 \\
\left\langle x_{i}\right\rangle & =0, \quad\left\langle\left(x_{i}\right)^{2}\right\rangle=\frac{1}{N}, \\
R_{B k} & =\frac{\boldsymbol{A} \cdot \boldsymbol{B}_{k}}{\|\boldsymbol{A}\|\left\|\boldsymbol{B}_{k}\right\|}, \quad q_{k k^{\prime}}=\frac{\boldsymbol{B}_{k} \cdot \boldsymbol{B}_{k^{\prime}}}{\left\|\boldsymbol{B}_{k}\right\|\left\|\boldsymbol{B}_{k^{\prime}}\right\|}, \\
R_{J} & =\frac{\boldsymbol{A} \cdot \boldsymbol{J}}{\|\boldsymbol{A}\|\|\boldsymbol{J}\|}, \quad R_{B k J}=\frac{\boldsymbol{B}_{k} \cdot \boldsymbol{J}}{\left\|\boldsymbol{B}_{k}\right\|\|\boldsymbol{J}\|},
\end{aligned}
$$

where $\langle\cdot\rangle$ denotes a mean.

Figure 1 illustrates the relationship among true teacher $\boldsymbol{A}$, ensemble teachers $\boldsymbol{B}_{k}$, student $\boldsymbol{J}$ and direction cosines $q_{k k^{\prime}}, R_{B k}, R_{J}$ and $R_{B k J}$.

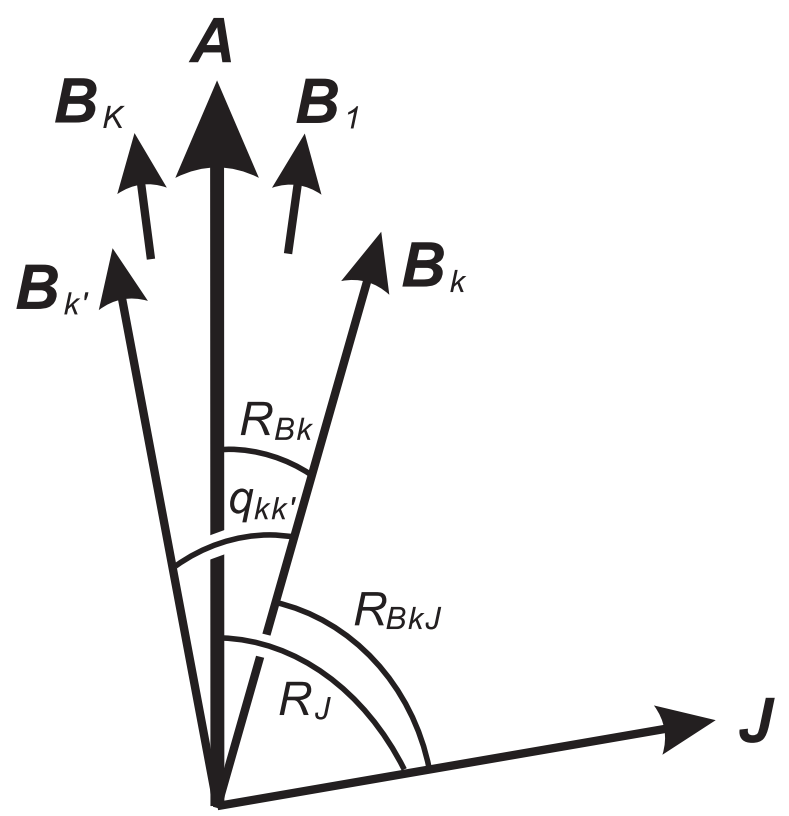

Fig. 1. True teacher $\mathbf{A}$, ensemble teachers $\mathbf{B}_{k}$ and student $\mathbf{J}$. $q_{k k^{\prime}}, R_{J}, R_{B k}$ and $R_{B k J}$ are direction cosines.

In this paper, the thermodynamic limit $N \rightarrow \infty$ is also treated. Therefore,

$$
\|\boldsymbol{A}\|=\sqrt{N}, \quad\left\|\boldsymbol{B}_{k}\right\|=\sqrt{N}, \quad\left\|\boldsymbol{J}^{0}\right\|=\sqrt{N}, \quad\|\boldsymbol{x}\|=1 .
$$

Generally, norm $\|\boldsymbol{J}\|$ of the student changes as time step proceeds. Therefore, ratios $l^{m}$ of the norm to $\sqrt{N}$ are introduced and called the length of the student. That is, $\left\|\boldsymbol{J}^{m}\right\|=l^{m} \sqrt{N}$, where $m$ denotes the time step.

The outputs of the true teacher, the ensemble teachers, and the student are $y^{m}+n_{A}^{m}, v_{k}^{m}+n_{B k}^{m}$ and $u^{m} l^{m}+n_{J}^{m}$, respectively. Here,

$$
\begin{aligned}
y^{m} & =\boldsymbol{A} \cdot \boldsymbol{x}^{m}, \\
v_{k}^{m} & =\boldsymbol{B}_{k} \cdot \boldsymbol{x}^{m}, \\
u^{m} l^{m} & =\boldsymbol{J}^{m} \cdot \boldsymbol{x}^{m}, \\
n_{A}^{m} & \sim \mathcal{N}\left(0, \sigma_{A}^{2}\right), \\
n_{B k}^{m} & \sim \mathcal{N}\left(0, \sigma_{B k}^{2}\right), \\
n_{J}^{m} & \sim \mathcal{N}\left(0, \sigma_{J}^{2}\right) .
\end{aligned}
$$

That is, the outputs of the true teacher, the ensemble teachers and the student include independent Gaussian noises with variances of $\sigma_{A}^{2}, \sigma_{B k}^{2}$, and $\sigma_{J}^{2}$, respectively. Then, $y^{m}, v_{k}^{m}$, and $u^{m}$ of Eqs. (8)-(10) obey Gaussian distributions with a mean of zero and variance unity.

Let us define the error $\epsilon_{B k}$ between true teacher $\boldsymbol{A}$ and each member $\boldsymbol{B}_{k}$ of the ensemble teachers by the squared errors of their outputs:

$$
\epsilon_{B k}^{m} \equiv \frac{1}{2}\left(y^{m}+n_{A}^{m}-v_{k}^{m}-n_{B k}^{m}\right)^{2} .
$$

In the same manner, let us define error $\epsilon_{B k J}$ between each member $\boldsymbol{B}_{k}$ of the ensemble teachers and student $\boldsymbol{J}$ by the squared errors of their outputs:

$$
\epsilon_{B k J}^{m} \equiv \frac{1}{2}\left(v_{k}^{m}+n_{B k}^{m}-u^{m} l^{m}-n_{J}^{m}\right)^{2} .
$$

Student $\boldsymbol{J}$ adopts the gradient method as a learning rule and uses input $\boldsymbol{x}$ and an output of one of the $K$ ensemble teachers $\boldsymbol{B}_{k}$ in turn or randomly for updates. That is,

$$
\begin{aligned}
\boldsymbol{J}^{m+1} & =\boldsymbol{J}^{m}-\eta \frac{\partial \epsilon_{B k J}^{m}}{\partial \boldsymbol{J}^{m}} \\
& =\boldsymbol{J}^{m}+\eta\left(v_{k}^{m}+n_{B k}^{m}-u^{m} l^{m}-n_{J}^{m}\right) \boldsymbol{x}^{m}
\end{aligned}
$$

where $\eta$ denotes the learning rate of the student and is a constant number. In cases where the student uses $K$ ensemble teachers in turn, $k=\bmod (m, K)+1$. Here, $\bmod (m, K)$ denotes the remainder of $m$ divided by $K$. On the other hand, in random cases, $k$ is a uniform random integer that takes one of $1,2, \ldots, K$.

Generalizing the learning rules, Eq. (17) can be expressed as

$$
\begin{aligned}
\boldsymbol{J}^{m+1} & =\boldsymbol{J}^{m}+f_{k} \boldsymbol{x}^{m} \\
& =\boldsymbol{J}^{m}+f\left(v_{k}^{m}+n_{B k}^{m}, u^{m} l^{m}+n_{J}^{m}\right) \boldsymbol{x}^{m}
\end{aligned}
$$

where $f$ denotes a function that represents the update amount and is determined by the learning rule.

In addition, let us define the error $\epsilon_{J}$ between true teacher $\boldsymbol{A}$ and student $\boldsymbol{J}$ by the squared error of their outputs:

$$
\epsilon_{J}^{m} \equiv \frac{1}{2}\left(y^{m}+n_{A}^{m}-u^{m} l^{m}-n_{J}^{m}\right)^{2}
$$

\section{THEORY}

\section{A. Generalization error}

A goal of statistical learning theory is to theoretically obtain generalization errors. Since generalization error is the mean of errors for the true teacher over the distribution of new input and noises, generalization error $\epsilon_{B k g}$ of each 
member $\boldsymbol{B}_{k}$ of the ensemble teachers and $\epsilon_{J g}$ of student $\boldsymbol{J}$ are calculated as follows. Superscripts $m$, which represent the time step, are omitted for simplicity unless stated otherwise.

$$
\begin{aligned}
\epsilon_{B k g}= & \int d \boldsymbol{x} d n_{A} d n_{B k} P\left(\boldsymbol{x}, n_{A}, n_{B k}\right) \epsilon_{B k} \\
= & \int d y d v_{k} d n_{A} d n_{B k} P\left(y, v_{k}, n_{A}, n_{B k}\right) \\
& \times \frac{1}{2}\left(y+n_{A}-v_{k}-n_{B k}\right)^{2} \\
= & \frac{1}{2}\left(-2 R_{B k}+2+\sigma_{A}^{2}+\sigma_{B k}^{2}\right) \\
= & \int d \boldsymbol{x} d n_{A} d n_{J} P\left(\boldsymbol{x}, n_{A}, n_{J}\right) \epsilon_{J} \\
\epsilon_{J g} & \int d y d u d n_{A} d n_{J} P\left(y, u, n_{A}, n_{J}\right) \\
& \times \frac{1}{2}\left(y+n_{A}-u l-n_{J}\right)^{2} \\
= & \frac{1}{2}\left(-2 R_{J} l+l^{2}+1+\sigma_{A}^{2}+\sigma_{J}^{2}\right) .
\end{aligned}
$$

Here, integrations have been executed using the following: $y, v_{k}$ and $u$ obey $\mathcal{N}(0,1)$. The covariance between $y$ and $v_{k}$ is $R_{B k}$, that between $v_{k}$ and $u$ is $R_{B k J}$, and that between $y$ and $u$ is $R_{J}$. All $n_{A}, n_{B k}$, and $n_{J}$ are independent from other probabilistic variables.

\section{B. Differential equations for order parameters and their analytical solutions}

To simplify the analysis, the following auxiliary order parameters are introduced:

$$
\begin{aligned}
r_{J} & \equiv R_{J} l \\
r_{B k J} & \equiv R_{B k J} l .
\end{aligned}
$$

Simultaneous differential equations in deterministic forms [8], which describe the dynamical behaviors of order parameters, have been obtained based on self-averaging in the thermodynamic limits as follows:

$$
\begin{aligned}
\frac{d r_{B k J}}{d t} & =\frac{1}{K} \sum_{k^{\prime}=1}^{K}\left\langle f_{k^{\prime}} v_{k}\right\rangle, \\
\frac{d r_{J}}{d t} & =\frac{1}{K} \sum_{k=1}^{K}\left\langle f_{k} y\right\rangle, \\
\frac{d l}{d t} & =\frac{1}{K} \sum_{k=1}^{K}\left(\left\langle f_{k} u\right\rangle+\frac{1}{2 l}\left\langle f_{k}^{2}\right\rangle\right) .
\end{aligned}
$$

Here, dimension $N$ has been treated to be sufficiently greater than the number of ensemble teachers $K$. Time is defined by $t=m / N$, that is, time step $m$ normalized by dimension $N$. Note that the above differential equations are identical whether the $K$ ensemble teachers are used in turn or randomly.

Since linear perceptrons are treated in this paper, the sample averages that appeared in the above equations can be easily calculated as follows:

$$
\begin{aligned}
&\left\langle f_{k} u\right\rangle=\eta\left(\frac{r_{B k J}}{l}-l\right), \\
&\left\langle f_{k}^{2}\right\rangle=\eta^{2}\left(l^{2}-2 r_{B k J}+1+\sigma_{B k}^{2}+\sigma_{J}^{2}\right), \\
&\left\langle f_{k} y\right\rangle=\eta\left(R_{B k}-r_{J}\right), \\
& \frac{1}{K} \sum_{k^{\prime}=1}^{K}\left\langle f_{k^{\prime}} v_{k}\right\rangle=\eta\left(-r_{B k J}+\frac{1}{K} \sum_{k^{\prime}=1}^{K} q_{k k^{\prime}}\right) .
\end{aligned}
$$

Since all components $A_{i}, J_{i}^{0}$ of true teacher $\boldsymbol{A}$, and the initial student $\boldsymbol{J}^{0}$ are drawn from $\mathcal{N}(0,1)$ independently and because the thermodynamic limit $N \rightarrow \infty$ is also treated, they are orthogonal to each other in the initial state. That is,

$$
R_{J}=0 \text { when } t=0 \text {. }
$$

In addition,

$$
l=1 \text { when } t=0 .
$$

By using Eqs. (32)-(37), simultaneous differential equations Eqs. (29)-(31) can be solved analytically as follows:

$$
\begin{aligned}
r_{B k J} & =\frac{1}{K} \sum_{k^{\prime}=1}^{K} q_{k k^{\prime}}\left(1-e^{-\eta t}\right) \\
r_{J} & =\frac{1}{K} \sum_{k=1}^{K} R_{B k}\left(1-e^{-\eta t}\right) \\
l^{2} & =\frac{1}{2-\eta}\left[2(1-\eta) \bar{q}+\eta\left(1+\bar{\sigma}_{B}^{2}+\sigma_{J}^{2}\right)\right] \\
& +\left[1+\frac{1}{2-\eta}\left(\eta\left(1+\bar{\sigma}_{B}^{2}+\sigma_{J}^{2}\right)-2 \bar{q}\right)\right] e^{\eta(\eta-2) t} \\
& -2 \bar{q} e^{-\eta t}
\end{aligned}
$$

where

$$
\begin{aligned}
\bar{q} & =\frac{1}{K^{2}} \sum_{k=1}^{K} \sum_{k^{\prime}=1}^{K} q_{k k^{\prime}}, \\
\bar{\sigma}_{B}^{2} & =\frac{1}{K} \sum_{k=1}^{K} \sigma_{B k}^{2} .
\end{aligned}
$$

\section{RESUlTS AND Discussion}

In this section, we treat the case where direction cosines $R_{B k}$ between the ensemble teachers and the true teacher, direction cosines $q_{k k^{\prime}}$ among the ensemble teachers and variances $\sigma_{B k}^{2}$ of the noises of ensemble teachers are uniform. That is,

$$
\begin{aligned}
R_{B k} & =R_{B}, \quad k=1, \ldots, K, \\
q_{k k^{\prime}} & = \begin{cases}q, & k \neq k^{\prime}, \\
1, & k=k^{\prime},\end{cases} \\
\sigma_{B k}^{2} & =\sigma_{B}^{2} .
\end{aligned}
$$

In this case, Eqs. (41) and (42) are expressed as

$$
\begin{aligned}
\bar{q} & =q+\frac{1-q}{K}, \\
\bar{\sigma}_{B}^{2} & =\sigma_{B}^{2} .
\end{aligned}
$$


The dynamical behaviors of generalization errors $\epsilon_{J g}$ have been analytically obtained by solving Eqs. (26), (27) and (38)-(47). Figure 2 shows the analytical results and the corresponding simulation results, where $N=2000$. In computer simulations, $K$ ensemble teachers are used in turn. $\epsilon_{J g}$ was obtained by averaging the squared errors for $10^{4}$ random inputs at each time step. Generalization error $\epsilon_{B g}$ of one of the ensemble teachers is also shown. The dynamical behaviors of $R$ and $l$ are shown in Fig. 3 .

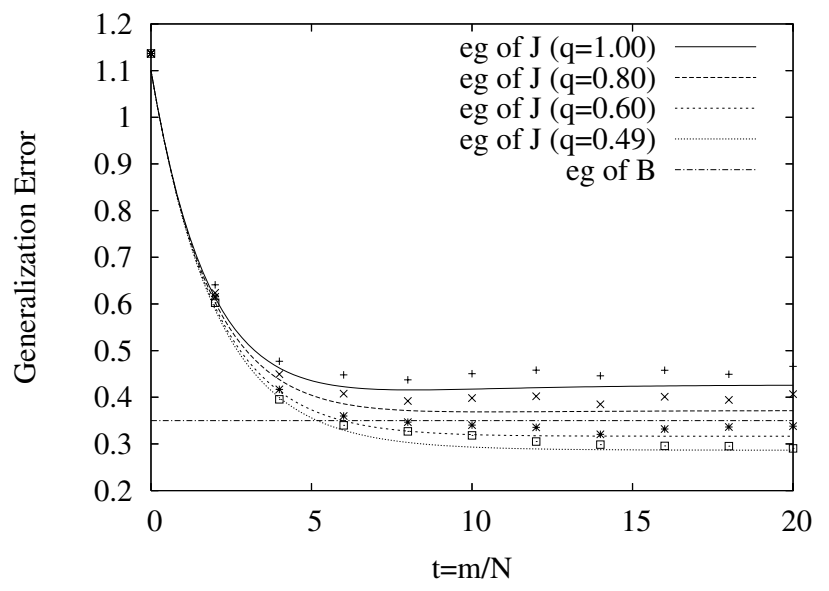

Fig. 2. Dynamical behaviors of generalization errors $\epsilon_{J g}$. Theory and computer simulations. Conditions other than $q$ are $\eta=0.3, K=3, R_{B}=$ $0.7, \sigma_{A}^{2}=0.0, \sigma_{B}^{2}=0.1$ and $\sigma_{J}^{2}=0.2$.

In these figures, the curves represent theoretical results. The dots represent simulation results. Conditions other than $q$ are common: $\eta=0.3, K=3, R_{B}=0.7, \sigma_{A}^{2}=0.0, \sigma_{B}^{2}=$ 0.1 and $\sigma_{J}^{2}=0.2$. Figure 2 shows that the smaller $q$ is, that is, the richer the variety of the ensemble teachers is, the smaller generalization error $\epsilon_{J g}$ of the student is. Especially in the cases of $q=0.6$ and $q=0.49$, the generalization error of the student becomes smaller than a member of the ensemble teachers after $t \approx 5$. This means that the student in this model can become cleverer than each member of the ensemble teachers even though the student only uses the input-output pairs of members of the ensemble teachers. Figure 3 shows that the larger the variety of the ensemble teachers is, the larger direction cosine $R_{J}$ is and the smaller length $l$ of the student is. The reason why minimum value 0.49 of $q$ is taken as the squared value of $R_{B}=0.7$ in Figs. 2 and 3 is described later.

In Figs. 2 and $3, \epsilon_{J g}, R_{J}$ and $l$ almost seem to reach a steady state by $t=20$. The macroscopic behaviors of $t \rightarrow \infty$ can be understood theoretically since the order parameters have been obtained analytically. Focusing on the signs of the powers of the exponential functions in Eqs. (38)-(40), we can see that $\epsilon_{J g}$ and $l$ diverge if $\eta<0$ or $\eta>2$. The steady state values of $r_{B k J}, r_{J}$ and $l^{2}$ in the case of $0<\eta<2$ can be easily obtained by substituting $t \rightarrow \infty$ in Eqs. (38)-(40)

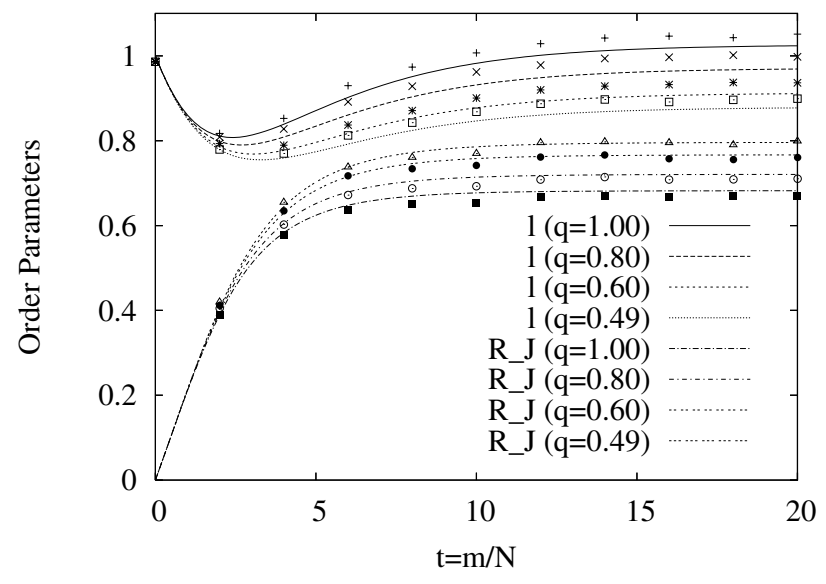

Fig. 3. Dynamical behaviors of $R_{J}$ and $l$. Theory and computer simulations. Conditions other than $q$ are $\eta=0.3, K=3, R_{B}=0.7, \sigma_{A}^{2}=$ $0.0, \sigma_{B}^{2}=0.1$ and $\sigma_{J}^{2}=0.2$.

as follows:

$$
\begin{aligned}
r_{B k J} & \rightarrow q+\frac{1-q}{K} \\
r_{J} & \rightarrow R_{B} \\
l^{2} & \rightarrow \frac{1}{2-\eta}\left(2(1-\eta)\left(q+\frac{1-q}{K}\right)\right. \\
& \left.+\eta\left(1+\sigma_{B}^{2}+\sigma_{J}^{2}\right)\right) \\
& =q+\frac{1-q}{K} \\
& +\frac{\eta}{2-\eta}\left(\frac{(1-q)(K-1)}{K}+\sigma_{B}^{2}+\sigma_{J}^{2}\right)
\end{aligned}
$$

Equations (26), (27) and (48)-(51) show the following: in the case of $\eta=1$, the steady value of length $l$ is independent from the number $K$ of teachers and direction cosine $q$ among the ensemble teachers. Therefore, the steady value of generalization error $\epsilon_{J g}$ and direction cosine $R_{J}$ are independent from $K$ and $q$ in this case. In the case of $0<\eta<1$, the smaller $q$ is or the larger $K$ is, the smaller the steady values of $l$ and $\epsilon_{J g}$ are and the larger the steady value of $R_{J}$ is. In the case of $1<\eta<2$, on the contrary, the smaller $q$ is or the larger $K$ is, the larger the steady values of $l$ and $\epsilon_{J g}$ are and the smaller the steady value of $R_{J}$ is. That is, in the case of $\eta<1$, the more teachers exist and the richer the variety of teachers is, the cleverer the student can become. On the contrary, in the case of $\eta>1$, the number of teachers should be small and the variety of teachers should be low for the student to become clever.

In the right hand side of Eq. (51), since the second and the third terms are positive, the steady value of $l$ is larger than $\sqrt{q}$. In addition, since $l \rightarrow \sqrt{q}$ in the limit of $\eta \rightarrow 0$ and $K \rightarrow \infty$, Eqs. (27) and (49) show $R_{J} \rightarrow R_{B} / \sqrt{q}$. On the other hand, when $S$ and $\boldsymbol{T}$ are generated independently under conditions where the direction cosine between $S$ and $\boldsymbol{P}$ and between $\boldsymbol{T}$ and $\boldsymbol{P}$ are both $R_{0}$, where $\boldsymbol{S}, \boldsymbol{T}$ and $\boldsymbol{P}$ are high dimensional vectors, the direction cosine between $\boldsymbol{S}$ and $\boldsymbol{T}$ is $q_{0}=R_{0}^{2}$. Therefore, if ensemble teachers have enough 
variety that they have been generated independently under the condition that all direction cosines between ensemble teachers and the true teacher are $R_{B}, R_{B} / \sqrt{q}=1$, then direction cosine $R_{J}$ between the student and the true teacher approaches unity regardless of the variances of noises in the limit of $\eta \rightarrow 0$ and $K \rightarrow \infty$.

Figures 4-7 show the relationships between the learning rate $\eta$ and $\epsilon_{J g}, R_{J}$. In Figs 4 and $5, K=3$ and is fixed. In Figs 6 and 7, $q=0.49$ and is fixed. Conditions other than $K$ and $q$ are $\sigma_{A}^{2}=\sigma_{B}^{2}=\sigma_{J}^{2}=0.0$ and $R_{B}=0.7$. Computer simulations have been executed using $\eta=0.3,0.6,1.0,1.4$ and 1.7. The values on $t=20$ are plotted for the simulations and considered to have already reached a steady state.

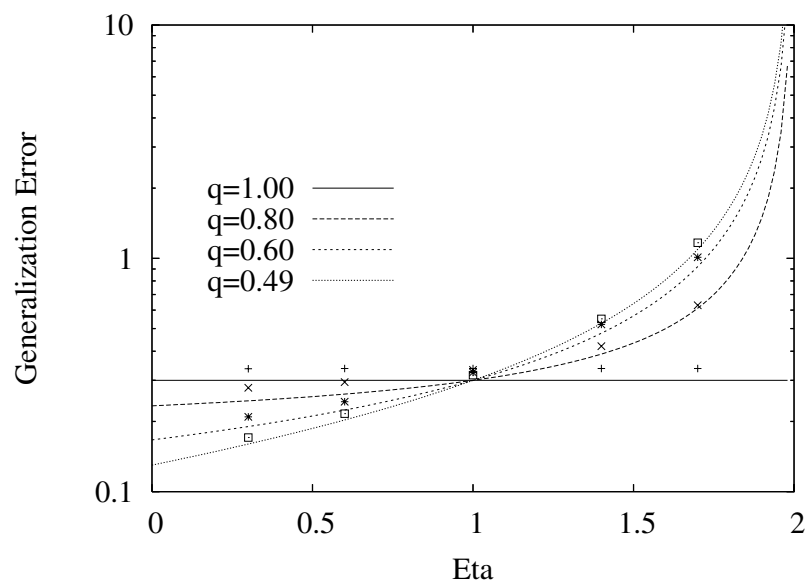

Fig. 4. Steady value of generalization error $\epsilon_{J g}$ in the case of $K=3$. Theory and computer simulations. Conditions other than $K$ and $q$ are $\sigma_{A}^{2}=$ $\sigma_{B}^{2}=\sigma_{J}^{2}=0.0$ and $R_{B}=0.7$.

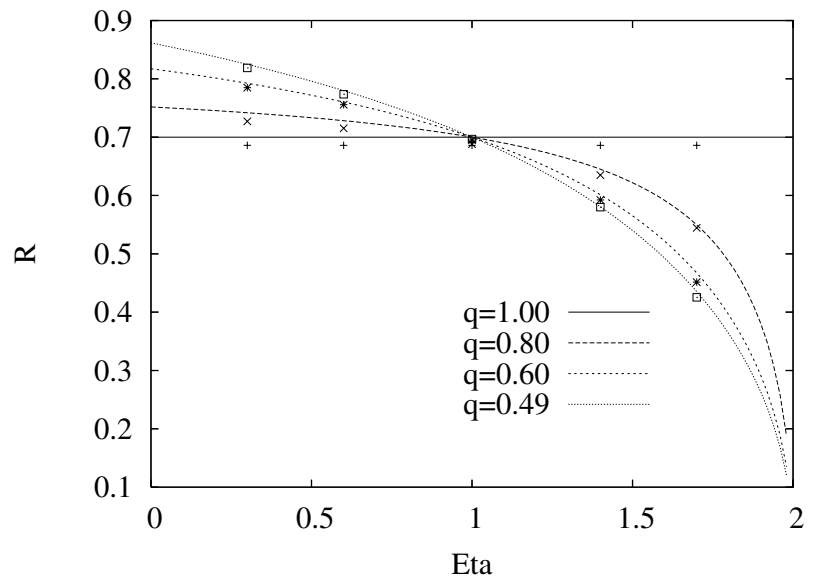

Fig. 5. Steady value of direction cosine $R_{J}$ in the case of $K=3$. Theory and computer simulations. Conditions other than $K$ and $q$ are $\sigma_{A}^{2}=\sigma_{B}^{2}=$ $\sigma_{J}^{2}=0.0$ and $R_{B}=0.7$.

These figures show the following: the smaller learning rate $\eta$ is, the smaller generalization error $\epsilon_{J g}$ is and the larger direction cosine $R_{J}$ is. Needless to say, when $\eta$ is small,

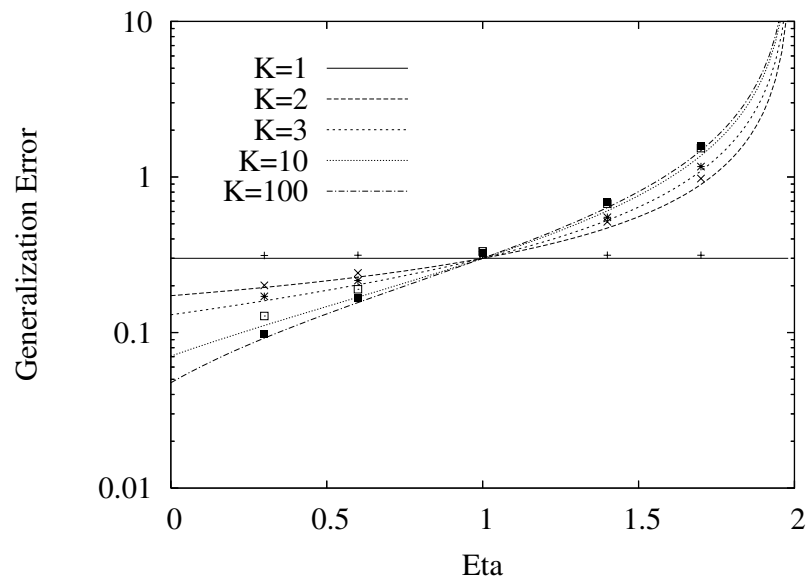

Fig. 6. Steady value of generalization error $\epsilon_{J g}$ in the case of $q=0.49$. Theory and computer simulations. Conditions other than $K$ and $q$ are $\sigma_{A}^{2}=$ $\sigma_{B}^{2}=\sigma_{J}^{2}=0.0$ and $R_{B}=0.7$.

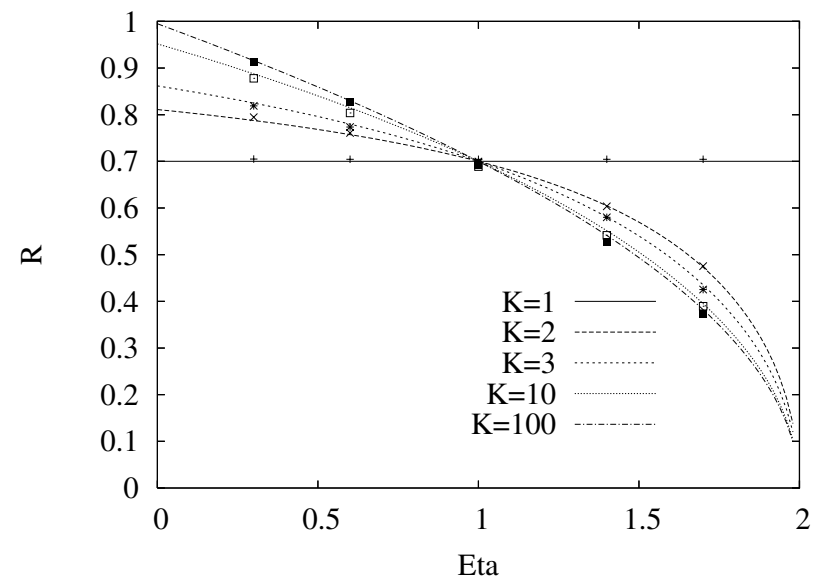

Fig. 7. Steady value of direction cosine $R_{J}$ in the case of $q=0.49$. Theory and computer simulations. Conditions other than $K$ and $q$ are $\sigma_{A}^{2}=\sigma_{B}^{2}=$ $\sigma_{J}^{2}=0.0$ and $R_{B}=0.7$.

learning is slow. Therefore, residual generalization error and learning speed are in a relationship of tradeoff. The phase transition in which $\epsilon_{J g}$ diverges and $R_{J}$ becomes zero at $\eta=2$ is shown. In the case of $\eta<1$, the larger $K$ is or the smaller $q$ is, that is, the richer the variety of ensemble teachers is, the smaller $\epsilon_{J g}$ is and the larger $R_{J}$ is. On the contrary, the properties are completely reversed in the case of $\eta>1$.

\section{CONClusion}

We analyzed the generalization performance of a student in a model composed of linear perceptrons: a true teacher, ensemble teachers, and the student. The generalization error of the student was analytically calculated using statistical mechanics in the framework of online learning, proving that when student's learning rate satisfies $\eta<1$, the larger the number $K$ is and the more variety the ensemble teachers have, the smaller the generalization error is. On the other 
hand, when $\eta>1$, the properties are completely reversed. If the variety of ensemble teachers is rich enough, the direction cosine between the true teacher and the student becomes unity in the limit of $\eta \rightarrow 0$ and $K \rightarrow \infty$.

\section{ACKNOWLEDGMENTS}

This research was partially supported by the Ministry of Education, Culture, Sports, Science, and Technology of Japan, with Grants-in-Aid for Scientific Research 14084212, 15500151 and 16500093.

\section{REFERENCES}

[1] D. Saad, (ed.), On-line Learning in Neural Networks, Cambridge: Cambridge University Press, 1998.

[2] Y. Freund and R. E. Schapire, "A short introduction to boosting," Journal of Japanese Society for Artificial Intelligence, 14, pp.771-780, 1999. [in Japanese, translation by N. Abe]

[3] A. Krogh and P. Sollich, "Statistical mechanics of ensemble learning, Phys. Rev. E 55, pp.811-825, 1997.

[4] R. Urbanczik, "Online learning with ensembles, Phys. Rev. E 62, pp.1448-1451, 2000.

[5] K. Hara and M. Okada, "Ensemble learning of linear perceptron; Online learning theory”, J. Phys. Soc. Jpn. 74, pp.2966-2972, 2005.

[6] S. Miyoshi, K. Hara and M. Okada, "Analysis of ensemble learning using simple perceptrons based on online learning theory", Phys. Rev. E 71, 036116, 2005.

[7] S. Miyoshi and M. Okada: "Analysis of on-line learning when a moving teacher goes around a true teacher", J. Phys. Soc. Jpn. 75, 2005. in press.

[8] H. Nishimori, Statistical Physics of Spin Glasses and Information Processing: An Introduction, Oxford: Oxford University Press, 2001. 